\title{
Renormalized field theory of polymer solutions. I. Scaling laws
}

\author{
Lothar Schäfer \\ Institute for Theoretical Physics, University of Heidelberg, 6900 Heidelberg, West Germany
}

Thomas A. Witten, Jr.

Randall Laboratory of Physics, University of Michigan, Ann Arbor, Michigan 48109

(Received 27 July 1976)

\begin{abstract}
The large scale spatial correlations in a dilute solution of long chain molecules are dominated by excluded volume effects. We extend the field-theoretic methods of Des Cloizeaux and De Gennes to describe these correlations. We derive general scaling laws which the experimentally measured correlation functions must obey. The dependence on (scattering) wave number, concentration, and chain length is thereby reduced to a function of two variables. This function may moreover be calculated via well-studied approximations, leaving only an excluded volume parameter and a microscopic length parameter. Using the scaling law, we derive several experimentally observed power laws for parameters such as the radius of gyration, including limiting behavior for small overlap (independent chains), large overlap, and large wave vector.
\end{abstract}

\section{INTRODUCTION}

The properties of long-chain polymers in solution are often found experimentally to obey simple power laws as functions of chain length and concentration. ${ }^{12}$ For instance, the radius of gyration $R$ of an isolated chain appears to vary with the number of monomers $N$ according to

$$
R=K N^{\nu},
$$

where $\nu \approx 0.6$ and $K$ is an arbitrary constant. The exponent $\nu$ is believed to take the same value independent of the nature of the chain or the solvent. The only requirements are that the chains not overlap, that the temperature be high enough to assure good solvation, ${ }^{1 b}$ and that the number of monomers be sufficiently large. Behavior similar to this has also been observed in computer studies of the self-avoiding random walk. ${ }^{2}$

Usually the explanation of these phenomena follows ideas of Flory. ${ }^{3}$ Recently, however, DeGennes ${ }^{4}$ and Des Cloizeaux ${ }^{5}$ have proposed a new theory to explain these "excluded volume" effects. Their treatment shows that certain correlations in a solution of chains may be calculated using a classical field theory of the same type used to study phase-transition models like the Ising model. When applied to phase transitions, the field theory explains the origin of critical exponents and other critical phenomena. ${ }^{8}$ Applied to the polymer system, the field theory explains the occurrence of power laws like Eq. (1). In particular, the exponent $\dot{\nu}$ defined above emerges as a critical exponent.

The work of Des Cloizeaux and of Emery ${ }^{7}$ makes it clear that the excluded volume problem is closely related to the random medium problem, ${ }^{8}$ the percolation problem, ${ }^{9}$ and the spin glass problem. ${ }^{10 a}$

In the present paper we extend the work of Des Cloizeaux and DeGennes. Their work shows how the field theory may be used to calculate the correlations between the end points of chains. Our work shows in addition how to calculate the correlations between arbitrary points of the chain(s). This is important because these latter correlations are the only type measurable in current experiments. Further, there is currently some disagreement ${ }^{10 b, 11}$ over what form the experimental correlations should take. In the following section we define the correlations to be studied in terms of a suitable model of the polymer system due to Edwards. ${ }^{12}$ Then we display the field theory and explain how it is used to calculate polymer correlations. We indicate how the field theory may be renormalized and how scaling laws for the correlations emerge from this renormalized field theory. Finally, we use standard field theoretic results to discuss the behavior of our scaling functions for small and large wave number and concentration. (We use the short-distance expansion of $\mathrm{Wilson}^{13}$ and Kadanoff ${ }^{14}$ to discuss the large wave number behavior.) The theory contains only two adjustable parameters, and it thus predicts strong interrelations among the results of different experiments.

The behavior observed experimentally ${ }^{11}$ is consistent with these general predictions as far as the authors are aware. But more importantly, the machinery thus developed allows the exponent $\nu$ to be calculated approximately. ${ }^{15}$ Indeed, the same approximations may be used to calculate explicitly the correlations which are observed in scattering experiments. The most common approximation is the expansion in $(d-4)$, where $d$ is the dimension of space, as developed by Wilson ${ }^{6}$ and Brezin et al. ${ }^{16,17}$ Our calculations using this approximation are in progress.

At present our theory (as well as that of Des Cloizeaux and DeGennes) has a shortcoming that should be noted. We assume that the monomers are in a chemical equilibrium characterized by a monomer chemical potential; this entails a broad (poly-disperse) distribution of chain lengths. By contrast, most experiments are performed on a sharply peaked length distribution. Thus, some of our more detailed predictions may not apply to these experiments.

\section{POLYMER CORRELATIONS}

In describing the large-scale properties of our polymer solution, we do not expect the chemical details of the chain structure or of the interaction between mono- 
<smiles>[Mg][Mg][Te]</smiles>

mers to be important. Accordingly, we shall use a well-accepted model ${ }^{18}$ in which microscopic chemical features of the polymers are included in a schematic way. We consider a system of $m$ polymers in solution in a volume $\Omega$. Each chain $i$ consists of $N_{i}$ links of mean square length $2 d l^{2}$, and its configuration is determined by fixing the endpoints of all links $\left\{\mathbf{r}_{i}(0), \mathbf{r}_{i}(1)\right.$, $\left.\cdots, \mathbf{r}_{i}\left(N_{i}\right)\right\}$ (Fig. 1). The cohesive energy associated with each chain ("Gaussian chains") is

$$
H_{0}[N, \mathbf{r}(0), \cdots, \mathbf{r}(N)]=\frac{1}{4 l^{2}} \sum_{\lambda=1}^{N}[\mathbf{r}(\lambda)-\mathbf{r}(\lambda-1)]^{2} .
$$

The interaction energy of the system is due to a repulsive, ${ }^{19}$ short-range potential $V(x)$ between any two points of chains:

$$
H_{I}=\frac{1}{2} \sum_{i, i^{\prime}=1}^{m} \sum_{\lambda=1}^{N_{i}} \sum_{\lambda^{\prime}=1}^{N_{i}} V\left[\mathbf{r}_{i}(\lambda)-\mathbf{r}_{i} \cdot\left(\lambda^{\prime}\right)\right] .
$$

The total energy $H$ is thus

$$
H=\sum_{i=1}^{m} H_{0}\left[N_{i}, \mathbf{r}_{i}(0), \cdots, \mathbf{r}_{i}\left(N_{i}\right)\right]+H_{I}
$$

The partition function $Z\left(N_{1}, \cdots, N_{m}\right)$ of the chains is defined by

$$
\begin{aligned}
Z\left(N_{1}, \cdots, N_{m}\right) & =\int_{\Omega} d^{d}\left\{\left[\mathbf{r}_{i}(\lambda)\right]\right\} e^{-H} \\
& \equiv \int \prod_{\mu=1}^{m}\left(4 l^{2} \pi\right)^{-N_{\mu} d / 2} \prod_{\lambda_{\mu}=0}^{N_{\mu}} d^{d} \mathbf{r}_{\mu}\left(\lambda_{\mu}\right) e^{-H},
\end{aligned}
$$

where $d$ denotes the dimension of space and the temperature has been set to unity.

In the sequel we shall be concerned with field-theoretic techniques for calculating the correlations in this system. In order to apply these techniques we must transform to the grand canonical ensemble. We allow all numbers $m$ of chains but associate with each chain a weighting factor $l^{2} h_{0}^{2} / 2$. [This peculiar form of the weighting factor is motivated by the field theory. The chemical potential for chains is of course proportional to $-\log \left(l^{2} h_{0}^{2} / 2\right)$.] We allow all lengths of chains, but associate with each length $N_{i}$ a weighting factor $e^{-z N_{i}}$. (It is at this point that we confine ourselves to an ensemble of chains with equilibrium chain-length distribution.) The partition function for the system now becomes

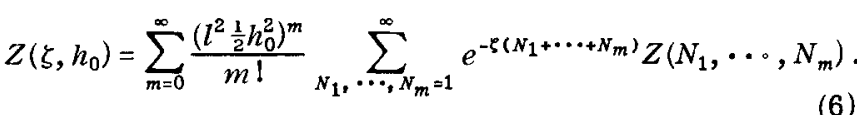

As usual, we may recover the expectation value of $m$ by differentiating $W \equiv\left[\log Z\left(\zeta, h_{0}\right)\right] / \Omega$ :

$$
\frac{\langle m\rangle}{\Omega}=\frac{\partial W}{\partial \ln \left(l^{2} h_{0}^{2} / 2\right)}=\frac{1}{2} h_{0} \frac{\partial W}{\partial h_{0}} .
$$

We may find the expectation value of the total length of all the chains by differentiating $W$ with respect to $\zeta$ :

$$
\frac{1}{\Omega}\left\langle\sum_{i=1}^{\infty} N_{i}\right\rangle=-\frac{\partial W}{\partial \zeta}
$$

The left-hand side of this equation is equal to $C_{l}$, the link concentration, while the quantity $\langle m\rangle / \Omega$ above is the concentration of chains $C_{p}$.

In order to define correlations, it is helpful to introduce the density functionals $\sigma_{i}(\mathbf{x})$ and $\rho_{i}(\mathbf{x})$. Thus, for example, the average density of endpoints of the first chain at location $\mathbf{x}$ can be expressed as

$$
\begin{aligned}
\left\langle\sigma_{1}(\mathbf{x})\right\rangle \equiv & \frac{1}{Z} \sum_{m=0}^{\infty} \frac{\left(l^{2} \frac{1}{2} h_{0}^{2}\right)^{m}}{m !} \sum_{N_{1} \cdots N_{m}=1}^{\infty} e^{-\mathcal{\xi}\left(N_{1} \cdots N_{m}\right)} \\
& \times \int_{\Omega} d^{d}\left\{\left[\mathbf{r}_{i}(\lambda)\right]\right\} e^{-H} \sigma_{1}(\mathbf{x})
\end{aligned}
$$

where

$$
\sigma_{i}(\mathbf{x}) \equiv\left\{\begin{array}{ll}
\delta\left[\mathbf{r}_{i}(0)-\mathbf{x}\right]+\delta\left[\mathbf{r}_{i}\left(N_{i}\right)-\mathbf{x}\right] & i \leq m \\
0 & i>m
\end{array} .\right.
$$

Evidently, the total density of endpoints $\left(2 C_{p}\right)$ can be expressed as $\langle\sigma(\mathbf{x})\rangle$, where

$$
\sigma(\mathbf{x}) \equiv \sum_{i=1}^{\infty} \sigma_{i}(\mathbf{x})
$$

In similar fashion, we may define a functional $\rho_{i}(\mathbf{x})$, whose expectation value gives the density of any point along the $i$ th chain at location $\mathbf{x}$ :

$$
\rho_{i}(\mathbf{x})= \begin{cases}\sum_{\lambda=1}^{N_{i}} \delta\left[\mathbf{r}_{i}(\lambda)-\mathbf{x}\right] & i \leq m \\ 0 & i>m\end{cases}
$$

The total density of links $C_{l}$ is then $\langle\rho(\mathbf{x})\rangle$, where $\rho(\mathbf{x}) \equiv \sum \rho_{i}(\mathbf{x})$.

Now the quantities observed in scattering experiments are related to correlations of the density at different locations. In an experiment where every monomer scatters equally, the amount of scattering at wave vector $k$ is proportional to $I_{0}(k)$, the Fourier transform of the cumulant $\langle\rho(\mathbf{x}) \rho(0)\rangle-\langle\rho(0)\rangle^{2}$ :

$$
\begin{aligned}
\delta\left(k+k^{\prime}\right) I_{0}(k) \equiv & (2 \pi)^{-2 d} \int d^{d} x d^{d} x^{\prime} e^{i k x} e^{i k^{\prime} x^{\prime}} \\
& \times\left[\left\langle\rho\left(x^{\prime}\right) \rho(x)\right\rangle-\langle\rho(0)\rangle^{2}\right] .
\end{aligned}
$$

Part of the intensity $I_{0}$ arises from correlations between different parts of the same chain. Defining $i_{a}$ as the scattering due to one chain only, we find

$$
\begin{aligned}
\delta\left(k+k^{p}\right) i_{a}(k) \equiv & \frac{1}{C_{p}}(2 \pi)^{-2 d} \int d^{d} x \int d^{d} x^{\prime} e^{i k x} e^{i k^{\prime} x^{\prime}} \\
& \times\left\langle\sum_{i=1}^{\infty} \rho_{i}(x) \rho_{i}\left(x^{\prime}\right)\right\rangle .
\end{aligned}
$$

This "autocorrelation intensity" $i_{a}$ can be measured by modifying the chains so that only a limiting small fraction of them cause scattering. In principle one may measure the correlations between the ends of chains by 
using chains in which only the ends cause scattering. If all the ends scattered equally, the intensity would be proportional to $I_{e}$, defined like $I_{0}$ with $\rho$ replaced by $\sigma$ everywhere. The autocorrelation part of $I_{e}$ is denoted by $i_{e a}$ and is defined like $i_{a}$ with $\rho$ replaced by $\sigma$. In principle it too is measurable.

We note that the grand canonical ensemble can be readily extended to include solutions of several species of chains. In that case we would have a separate $\zeta$ and $h_{0}$ parameter for each species.

\section{POLYMER FIELD THEORY}

Des Cloizeaux ${ }^{5}$ and DeGennes ${ }^{4}$ have shown that the Edwards model for polymers described above is mathematically equivalent to a certain classical field theory. At every point in space a vector $\varphi_{\alpha}(x)$ is defined, where $\alpha$ labels the $n$ vector components. The energy of a configuration is given by a Lagrangian of the form

$$
\begin{aligned}
L[\varphi]= & \frac{1}{2} \int d^{d} x \sum_{\alpha=1}^{n}\left\{\xi / l^{2}\left[\varphi_{\alpha}(x)\right]^{2}+\left|\nabla \varphi_{\alpha}(x)\right|^{2}\right\} \\
& +\frac{(2 \pi)^{d} l^{-4}}{8} \int d^{d} x \int d^{d} x^{\prime} \sum_{\alpha, \beta=1}^{n} \varphi_{\alpha}(x)^{2} V\left(x-x^{\prime}\right) \\
& \times \varphi_{\beta}\left(x^{\prime}\right)^{2}-\int d^{d} x h_{0} \varphi_{1}(x) .
\end{aligned}
$$

The partition function $Z_{n}\left(\zeta, h_{0}\right)$ of the field theory is as usual the weighted sum over all configurations:

$$
Z_{n}\left(\zeta, h_{0}\right) \equiv \int d\left[\varphi_{1}(x)\right] \cdots d\left[\varphi_{n}(x)\right] e^{-L[\varphi]} .
$$

The configuration integral is a sum over all functions $\varphi_{\alpha}(x)$ within the volume $\Omega$.

This field theory for $n=1,2$, and 3 has been widely used to represent bulk matter near a second-order phase transition. ${ }^{6,16}$ For example, to represent an isotropic ferromagnet, one takes $d=n=3$. Then $\zeta$ represents the temperature and $h_{0}$ the magnetic field. Des Cloizeaux and DeGennes have shown that in the limit of long chains the polymer model corresponds to an $n \rightarrow 0$ limit of this field theory. Thus $W\left(\zeta, h_{0}\right)$ defined above Eq. (7) is given by

$$
\Omega W\left(\zeta, h_{0}\right) \equiv \lim _{n \rightarrow 0}\left[\ln Z_{n}\left(\zeta, h_{0}\right)\right] .
$$

The limit requires that we give a meaning to $Z_{n}\left(\zeta, h_{0}\right)$ even when $n$ is not a natural number. This proves possible if we calculate $Z$ by the usual wick perturbation expansion (Feynman diagrams) in powers of $V(r) .{ }^{20}$ In

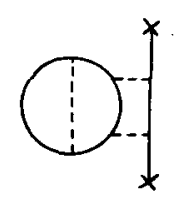

(a)

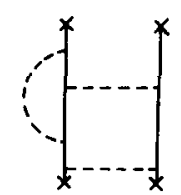

(b)
FIG. 2. Diag rams for $W_{n}\left(\zeta, h_{0}\right.$ (a) a diagram whose value is proportional to $n$; (b) a diagram independent of $n$, which contributes to $W\left(\xi, h_{0}\right)$ for $n=0$. Solid lines denote free propagators; broken lines denote factors of $-V\left(r^{\prime}-r\right) / 8$, where $r^{\prime}$ and $r$ are the endpoints of the broken lines. Crosses, $\times$, denote factors of $h_{0}$.

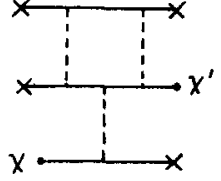

(a)

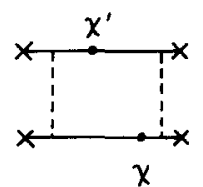

(c)

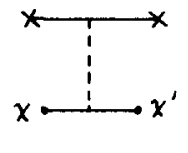

(b)

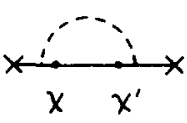

(d)
FIG. 3. Typical diagrams for various correlation functions. Diagrams $a$ and $b$ contribute to $I_{e}$; diagram $b$ also contrib-utes to $i_{e a}$. Diagrams $c$ and $d$ contribute to $I_{0} ;$ diagram $d$ also contributes to $i_{\boldsymbol{a}}$. that case each term in the expansion has an explicit polynomial dependence on $n$, and the meaning of the $n \rightarrow 0$ limit is unambiguous. ${ }^{21}$ The diagrammatic meaning of the $n \rightarrow 0$ limit is indicated in Fig. 2. Here we note that a general diagram contains loops of $\varphi_{\alpha}$ propagators, and after summing over the $n$ possible values of $\alpha$, each loop gives a contribution proportional to $n$. Thus the limit $n \rightarrow 0$ means precisely that all such loops are absent from the diagrams. The only propagator lines that remain are those which terminate at an $h_{0}$ vertex. It is suggestive to regard each diagram as a schematic picture of some of the configurations of the system. Each $\varphi$ propagator line represents a polymer, and the dotted interaction lines represent the repulsion acting between arbitrary points of the various polymers. A detailed examination ${ }^{5}$ bears out this interpretation.

The two-point correlation of $\varphi$ is found to be proportional to the intensity function $I_{e}$; indeed,

$$
\left\langle\sigma(x) \sigma\left(x^{\prime}\right)\right\rangle-\langle\sigma(0)\rangle^{2}=h_{0}^{2} \lim _{n \rightarrow 0}\left[\left\langle\varphi_{1}(x) \varphi_{1}\left(x^{\prime}\right)\right\rangle_{n}-\left\langle\varphi_{1}(0)\right\rangle_{n}^{2}\right],
$$

where again the limit $n \rightarrow 0$ is taken after the correlations have been computed from diagrams valid for general $n$. The diagrammatic representations of the $\varphi-\varphi$ correlations are illustrated in Fig. 3. In calculating the autocorrelation function we must guarantee that it is the ends of the same chain that scatter. Des Cloizeaux has shown that this can easily be done by asking for the correlations between field components $\varphi_{\alpha}(x)$ which do not couple to the field $h_{0}\left[\varphi_{2}(x)\right.$, for instance]. Properly normalized his result reads

$$
\left\langle\sum_{i=1}^{\infty} \sigma_{i}(x) \sigma_{i}\left(x^{\prime}\right)\right\rangle=h_{0}^{2} \lim _{n \rightarrow 0}\left\langle\varphi_{2}(x) \varphi_{2}\left(x^{\prime}\right)\right\rangle_{n} \text { 。 }
$$

Diagrammatically, one simply sums that subset of $\left\langle\varphi(x) \varphi\left(x^{\prime}\right)\right\rangle$ diagrams which have the two external endpoints $\varphi(x)$ and $\varphi\left(x^{\prime}\right)$ on the same line. On the other hand, in normal field theories with $n=2,3, \ldots$ the $\varphi_{2}-\varphi_{2}$ correlation is the transverse correlation.

We may now extend Des Cloizeaux's field theory to treat the experimentally observed intensities $I_{0}$ and $i_{a}$. To compute $I_{0}$ we require the Fourier transform of $\left\langle\rho(x) \rho\left(x^{\prime}\right)\right\rangle-\langle\rho\rangle^{2}$. This correlation is the (suitably normalized) sum over all configurations with the constraint that some point of a chain pass through $x$ and some point 
pass through $x^{\prime}$. To find the field theory analog of such a sum, it is suggestive to include just those Feynman diagrams for which some propagator (polymer) line passes through $x$ and some line passes through $x^{\prime}$ (Fig. 3). This is just the structure found in diagrams for $\left\langle\varphi^{2}(x) \varphi^{2}\left(x^{\prime}\right)\right\rangle$. The exact statement proves to be

$$
\begin{aligned}
& \left\langle\rho(x) \rho\left(x^{\prime}\right)\right\rangle-\langle\rho(0)\rangle^{2} \\
& \quad=l^{-4} \lim _{n \rightarrow 0}\left[\left\langle\frac{1}{2}|\varphi(x)|^{2} \frac{1}{2}\left|\varphi\left(x^{\prime}\right)\right|^{2}\right\rangle_{n}-\left\langle\frac{1}{2}|\varphi|^{2}\right\rangle_{n}^{2}\right],
\end{aligned}
$$

where $|\varphi|^{2}$ denotes $\sum_{\alpha} \varphi_{\alpha}^{2}$. The autocorrelation part $\left\langle\sum_{i} \rho_{i}(x) \rho_{i}\left(x^{\prime}\right)\right\rangle$ is given by that subset of diagrams for $\left\langle\frac{1}{2}|\varphi(x)|^{2} \frac{1}{2}\left|\varphi\left(x^{\prime}\right)\right|^{2}\right\rangle$ in which both $\varphi^{2}$ insertions occur on the same polymer line. We can find a closed-form expression for the autocorrelation by copying the procedure used experimentally. We introduce a second field $\varphi^{\prime}(x)$ into the Lagrangian to represent a second species of chain, whose concentration is taken to be very small. Then the autocorrelation of our original system can be expressed as $\left\langle\frac{1}{2}\left|\varphi^{\prime}(x)\right|^{2} \frac{1}{2}\left|\varphi^{\prime}\left(x^{\prime}\right)\right|^{2}\right\rangle$. We explain this more fully in Appendix A.

Equation (19) may be established rigorously by allowing the chemical potential $\zeta$ to depend on position $x$. We specify how this is done in Appendix A. Then since $\zeta(x)$ is conjugate to $\rho(x)$, we have

$$
\langle\rho(x) \rho(0)\rangle-\langle\rho(0)\rangle^{2}=\frac{\delta \Omega W}{\delta \zeta(x) \delta \zeta(0)} .
$$

However, the same derivative is defined in the field theory:

$$
\frac{\delta \Omega W_{n}}{\delta \zeta(x) \delta \zeta(0)}=l^{-4}\left[\left\langle\frac{1}{2}|\varphi(x)|^{2} \frac{1}{2}|\varphi(0)|^{2}\right\rangle_{n}-\left\langle\frac{1}{2}|\varphi(0)|^{2}\right\rangle_{n}^{2}\right]
$$

Now taking the limit $n \rightarrow 0$, we obtain Eq. (19). ${ }^{22}$

In diagrams for $W$ and the correlation functions $I$, certain basic pieces appear repeatedly; these special pieces are given the name "vertex functions." The vertex functions contain all the essential physics of the renormalization symmetry, and they are more manageable than the $W$ and $I$ functions themselves. A typical vertex function is the "four-point function" or "effective four-point coupling" $\Gamma^{(0,4)}\left(p_{1}, p_{2}, p_{3}, p_{4} ; \zeta\right)$. It is defined as the sum of all "irreducible" pieces of Feynman diagrams at chemical potential $\zeta$ having four vertices where propagator legs may be attached. Irreducible pieces are those which cannot be separated into disconnected parts by cutting a single propagator line. The wave numbers $p_{1} \cdots p_{4}$ are conjugate to the positions of the four vertices. For our discussion we must consider the general $\Gamma^{\left(L, N^{\prime}\right)}\left(q_{1} \cdots q_{L} ; p_{1} \cdots p_{N^{\prime}}, \zeta\right)$, which has $L \varphi^{2}$ vertices as well as $N^{\prime}$ places where propagator (polymer) lines may be attached. [The twopoint vertex $\Gamma^{(0,2)}\left(p_{1}-p, \zeta\right)$ contains an added noninteracting term $p^{2}+\zeta / l^{2}$.] Vertex functions are discussed in detail, for example, in Ref. 23.

We may construct $W\left(\zeta, h_{0}\right)$ and the intensity functions $I$ directly from algebraic combinations of the vertex functions. We introduce a parameter $M_{0}=\partial W\left(\zeta, h_{0}\right) / \partial h_{0}$ and we define $M_{0}$-dependent vertex functions as generating functions for our original $\Gamma$ 's:

$$
\begin{aligned}
& \Gamma_{b}^{\left(L, N^{\prime}\right)}\left(\left\{q_{i}\right\},\left\{p_{j}\right\}, \zeta, M_{0}\right) \\
& \quad \equiv \sum_{\nu=0}^{\infty} \frac{M_{0}^{\nu}}{\nu !} \Gamma_{b}^{\left(L, N^{\prime}+\nu\right)}\left(q_{1} \cdots q_{L} ; p_{1} \cdots p_{N^{\prime}}, 0, \cdots 0, \zeta\right) .
\end{aligned}
$$

Next we define the autocorrelation part $\Gamma_{b a}^{\left(L, N^{\prime}\right)}\left(\left\{q_{i}\right\}\right.$; $\left.\left\{p_{j}\right\}, \zeta, M_{0}\right)$ as that subset of the diagrams for $\Gamma_{b}^{\left(L, N^{\prime}\right)}$ $\left(\ldots, M_{0}\right)$ for which all the $L+N^{\prime}$ external vertices occur on the same polymer. line.

Now the connection of the $\Gamma_{b}^{\left(L, N^{*}\right)}$ to physical quantities is as follows. First the function $W$ is the Legendre transform of $\Gamma_{b}^{(0,0)}$ as derived in Ref. 23:

$$
W\left(\zeta, h_{0}\right)+\Gamma_{b}^{(0,0)}\left(\zeta, M_{0}\right)=M_{0} h_{0} \text {. }
$$

Using the properties of Legendre transformation we may re-express $C_{p}$ and $C_{l}$ in terms of derivatives of $\Gamma^{(0,0)}$. For example, $C_{p}$ is proportional to $\Gamma^{(0,1)}$ at zero momentum:

$$
C_{p}\left(=\frac{h_{0}}{2} \frac{\partial W}{\partial h_{0}}\right)=\frac{M_{0}}{2} \Gamma_{b}^{(0,1)}\left(\zeta, M_{0}\right)=\frac{h_{0} M_{0}}{2} 。
$$

Similarly,

$$
C_{l}=l^{-2} \Gamma_{b}^{(1,0)}\left(q=0, \zeta, M_{0}\right) .
$$

The end-point correlations can be shown to be inversely proportional to the two point function:

$$
\begin{aligned}
& I_{e}(p)=2 C_{p}^{2}\left[\frac{M_{0}^{2}}{2} \Gamma_{b}^{(0,2)}\left(p,-p, \zeta, M_{0}\right)\right]^{-1}, \\
& i_{e a}(p)=2 C_{p}\left[\frac{M_{0}^{2}}{2} \Gamma_{b a}^{(0,2)}\left(p,-p, \zeta, M_{0}\right)\right]^{-1} .
\end{aligned}
$$

The full density correlations are more complicated:

$$
\begin{aligned}
& I_{0}(p)=l^{-4}\left\{-\Gamma_{b}^{(2,0)}\left(p,-p, \zeta, M_{0}\right)+\frac{\left[\Gamma_{b}^{(1,1)}\left(p,-p, \zeta, M_{0}\right)\right]^{2}}{\Gamma_{b}^{(0,2)}\left(p,-p, \zeta, M_{0}\right)}\right\}, \\
& i_{a}(p)=\frac{1}{C_{p} l^{4}}\left\{-\Gamma_{b a}^{(2,0)}\left(p,-p, \zeta, M_{0}\right)+\frac{\left[\Gamma_{b a}^{(1,1)}\left(p,-p, \zeta, M_{0}\right)\right]^{2}}{\Gamma_{b a}^{(0,2)}\left(p,-p, \zeta, M_{0}\right)}\right\} .
\end{aligned}
$$

We illustrate the meaning of one of these expressions by the use of skeleton diagrams in Fig. 4.

\section{SCALING LAWS}

The renormalization symmetry of the vertex functions arises in the regime where the length scales of interest

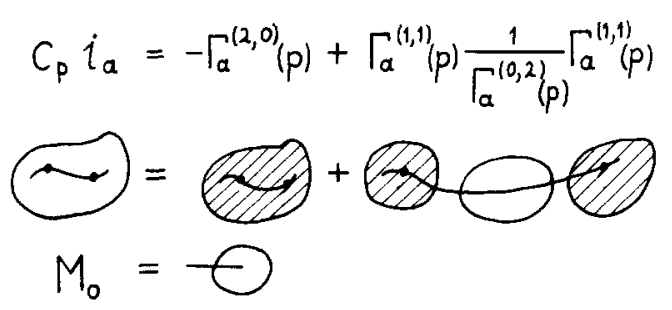

FIG. 4. Skeleton diagrams for $C_{p} i_{a}(p)$. Each $\varphi^{2}$ insertion and each external line is at wave number $p$. Open circles denote all diagrams with the indicated structure (with weighting factor $h_{0}$ at the end of each polymer line). Shaded circles denote irreducible diagrams (with weighting factor $M_{0}$ at the end of each polymer line). A diagrammatic representation for $M_{0}$ $\left(=\partial W / \partial h_{0}\right)$ is also shown. One readily verifies that a diagram belonging to the set on the left side must have the form of either the first or the second term on the right side. 
are much longer than both the length $l$ of a link and the range of $V(x)$. The physical phenomena of interest are not spoiled by taking $V(x)$ to be zero range:

$$
V(x)=\frac{g_{0}}{3(2 \pi)^{d}}(l \Lambda)^{\epsilon} l^{d} \delta^{d}(x) \quad \epsilon \equiv 4-d,
$$

where the wave number $\Lambda$ is defined below, and $g_{0}$ is a dimensionless coupling constant. This choice creates unphysical singularities due to integrations over large intermediate wave vectors (small distances) in the diagrams, which are regularized by introducing a cutoff wave vector $\Lambda$ as an upper bound in momentum (wave vector) integrations. There exist methods to subtract the singular parts of the diagrams in such a way that firstly the long-range behavior of interest remains unchanged, and secondly, the limit $\Lambda \rightarrow \infty$ can be taken in the subtracted theory. There exists a continuum of these "renormalization" methods, characterized by an arbitrary parameter $\mu$ with dimensions of a wave number. One then finds that the bare vertex functions are proportional to the renormalized ones, through two dimensionless factors $z$ and $z_{2}$, which depend only on $g_{0}$ and $\mu / \Lambda^{24}$ :

$$
\begin{aligned}
\Gamma_{b}^{\left(L, N^{\prime}\right)} & \left(q_{1} \cdots p_{N^{\prime}} ; \zeta, M_{0}, g_{0}, \Lambda\right) \\
= & \left(z_{2} / z\right)^{-L} z^{-N^{\prime} / 2} \Gamma_{R}^{\left(L, N^{\prime}\right)}\left(q_{1}, \cdots, p_{N} ; t, M, g^{*}, \mu\right) \\
& \times[1+\text { (corrections) }] .
\end{aligned}
$$

The $\Gamma_{R}$ 's can be calculated by a diagrammatic procedure similar to that for the $\Gamma_{b}$ 's, except that one replaces $M_{0}, \xi$, and $g_{0}$ by $M, t$, and $g^{*}$. The $M$ and $t$ prove to be linearly related to their bare counterparts:

$$
M=M_{0} z^{-1 / 2}, \quad t=\left(\zeta-\zeta_{c}\right) / l^{2}\left(z_{2} / z\right)^{-1} .
$$

The effective coupling constant $g^{*}$ is independent of $g_{0}$ 。 It depends only on the structure of the diagrams and the dimension of space. Thus in the $n$-component field theory, ${ }^{16}$

$$
g^{*}=\frac{(2 \pi)^{d} \Gamma(d / 2)}{2 \pi^{d / 2}} \frac{6}{n+8} \epsilon\left[1+\left(\frac{3(n+14)}{(n+8)^{2}}-\frac{1}{2}\right) \epsilon+\mathcal{O}\left(\epsilon^{2}\right)\right],
$$

and for the polymer field theory we set $n=0$. The "corrections" in Eq. (27) vanish in the limit $t / \Lambda^{2}, \mu / \Lambda$, $M / \Lambda^{1-\epsilon / 2} \rightarrow 0$, and we shall ignore them for the moment. The "critical chemical potential" $\zeta_{c}$ depends only on $g_{0}$. The renormalization of the autocorrelation parts $\left.\Gamma_{b a}^{(L, N},{ }^{\prime}\right)$ follows the same law with the same renormalization constants as does the full $\Gamma_{b}^{\left(L, N^{\prime}\right)}$.

Equation (27) implies that a change in $\mu$ can be compensated by a change of the scale factors $z_{2} / z$ and $z$, since the left side cannot depend on the renormalization parameter $\mu$. We may use the relation between $\mu$ and the $z$ 's to make explicit the dependence of the $\Gamma_{R}$ on one of its arguments. We can eliminate a second argument from the $\Gamma_{R}$ by using dimensional analysis. As a result, we can express $\Gamma_{R}^{\left(L, N^{\prime}\right)}$ in terms of a function $F^{\left(L, N^{\prime}\right)}$ having two fewer arguments:

$$
\begin{aligned}
& \Gamma_{R}^{\left(L, N^{\prime}\right)}\left(\left\{q_{i}\right\} ;\left\{p_{j}\right\}, t, M, \mu\right) \\
& \quad=\mu^{d-\left(N^{\circ} / 2\right)(d-2)-2 L}\left(g^{*}\right)^{N^{\prime} / 2-1} w^{N^{*} B-\nu d+L} F^{\left(L, N^{\prime}\right)}\left(\left\{Q_{i}\right\} ;\left\{P_{j}\right\} ; y\right),
\end{aligned}
$$

where $w$ and $y$ are dimensionless parameters defined by

$$
w \equiv\left[g * \frac{M^{2}}{\mu^{2-\epsilon}}\right]^{-1 / 2 \beta}, \quad y \equiv t w / \mu^{2},
$$

and $Q_{i}$ and $P_{j}$ are rescaled wave vectors:

$$
Q_{i} \equiv \frac{q_{i}}{\mu} w^{\nu}, \quad P_{j} \equiv \frac{p_{j}}{\mu} w^{\nu},
$$

The exponents $\nu$ and $\beta$ are critical exponents to be calculated from the renormalization factors. For example,

$$
\begin{aligned}
& \left.\tilde{\nu} \equiv \lim _{\Lambda \rightarrow \infty} \mu \frac{\theta}{\partial \mu}\right|_{g_{0}, \Lambda \text { fixed }} \ln \frac{z_{2}}{z}\left(g_{0}, \mu / \Lambda\right), \\
& \nu \equiv(\tilde{\nu}+2)^{-1}
\end{aligned}
$$

The quantities $\nu$ and $\tilde{\nu}$ are independent of $g_{0}$, since otherwise the $\Gamma_{R}$ 's would depend on $g_{0}$.

The scaling law of Eq. (28) expresses the renormalization symmetry in a concrete way. Only its structure arises from the symmetry; the symmetry says nothing about the "scaling functions" $F^{(L, N)}$ or about the values of the exponents $\nu$ and $\beta$. To determine these one must study the renormalized field theory in more detail. We merely note here that the same well-studied techniques used to estimate these objects in phase-transition problems can be applied directly to the polymer problem. Indeed, DeGennes ${ }^{4}$ has already estimated $\nu \approx 0.597$ using the technique of expanding in $(4-d)$. Since the exponents and scaling functions are purely properties of the renormalized theory, they are not affected by the chemical details of the polymer system: they are "universal." In the present work we shall limit ourselves to general consequences of the scaling law. We now deduce the scaling laws obeyed by the polymer variables as a consequence of $\mathrm{Eq}$. (28).

We could carry through the transformation to polymer variables directly in the renormalized formalism. We prefer however to transform back to the bare vertex functions in order to eliminate the unphysical parameter $\mu$. For this purpose we have to know the dependence of the renormalization factors on $\mu / \Lambda$. The method, which essentially consists in integrating relations like Eq. (29), is described in Ref. 25. The result for $z\left(g_{0}, \mu / \Lambda\right)$ derived there reads in our case

$$
z\left(g_{0}, \mu / \Lambda\right) \rightarrow A_{1}\left(g_{0}\right)(\mu / \Lambda)^{2-d+2 \beta / \nu} \quad \Lambda \gg \mu,
$$

where $A_{1}$ is a dimensionless constant of integration which may depend on $g_{0}$. The method can easily be modified to yield

$$
\frac{z_{2}}{z}\left(g_{0}, \mu / \Lambda\right)-A_{2}\left(g_{0}\right)(\mu / \Lambda)^{1 / \nu-2} \quad \Lambda \gg \mu .
$$

We now are in a position to describe the transformation ${ }^{5}$ from the renormalized variables $y, w$ to the (bare) polymer variables $C_{l}, C_{p}$ (or $C_{l}, N$ ). Equation (27) yields

$$
\Gamma_{b}^{(0,0)}\left(\zeta, M_{0}\right)=\Gamma_{R}^{(0,0)}(t, M)
$$

Using this, $C_{l}$ can be written in terms of the scaling function $F^{(0,0)}(y)$ : 


$$
\begin{aligned}
C_{l} & =\frac{\partial}{\partial \zeta} \Gamma_{b}^{(0,0)}=\frac{\partial t}{\partial \zeta} \mu^{-2} w \frac{\partial \Gamma_{R}^{(0,0)}}{\partial y} \\
& =S_{2}^{-1} \mu^{d}(\mu l)^{-1 / \nu} w^{1-\nu d}\left(g^{*}\right)^{-1} \frac{\partial F^{(0,0)}}{\partial y}
\end{aligned}
$$

where

$$
S_{2} \equiv A_{2}\left(g_{0}\right)(l \Lambda)^{2-1 / \nu} .
$$

The concentration $C_{p}$ can also be written in terms of $F^{(0,0)}$ :

$$
C_{p}=\frac{M_{0}}{2} \frac{\partial}{\partial M_{0}} \Gamma_{b}^{(0,0)}=\frac{M}{2} \frac{\partial}{\partial M} \Gamma_{R}^{(0,0)}=\frac{\mu^{d}}{2 g^{*}} w^{-\nu d} f(y),
$$

where

$$
f(y)=\frac{1}{\beta}\left(\nu d-y \frac{\partial}{\partial y}\right) F^{(0,0)}(y) .
$$

We introduce the "overlap" variable $s-a$ combination of $C_{p}$ and $N$ which depends only on the scaling variable $y$ :

$$
s \equiv \frac{1}{2} f(y)^{1-\nu d}\left(2 \frac{\partial F^{(0,0)}}{\partial y}\right)^{\nu d}=l^{t} g^{*} C_{p}\left(S_{2} N\right)^{\nu d} .
$$

By inverting this relation, one may obtain $y$ as a function of the overlap $s$. The variable $w$ and the momentum variables $Q$ can be expressed by

$$
\begin{aligned}
& w=N S_{2}(l \mu)^{1 / \nu} \frac{f(y)}{2\left(\partial F^{(0,0)} / \partial y\right)}, \\
& Q=q l\left(N S_{2}\right)^{\nu}\left(\frac{f(y)}{2\left(\partial F^{(0,0) / \partial y)}\right.}\right)^{\nu} .
\end{aligned}
$$

None of these equations involves the scale $A_{1}$. We will find below that the absence of $A_{1}$ is a general feature of our expressions.

In computing bare quantities the factors $\mu$ obviously have to cancel, and $\Lambda$ occurs only as a factor in $S_{2}$. Thus our theory involves only the excluded volume parameters $S_{2}$ and the microscopic length $l$, as does Flory's theory of excluded volume effects. ${ }^{26 a}$ (Further, as in Flory's theory, $l$ should vary only gradually with temperature。)

For readers familiar with the theory of magnetic phase transitions we include a plot showing the relation between the "magnetic" variables $(M, t)$ and the polymer variables $\left(C_{t}, N\right)$ using the mean-field $(d=4)$ values $f(y)$ $=y+1 / 6 ; \partial F^{(0,0)} / \partial y=1 / 2$ (Fig. 5).

We can now write the scaling laws for the scattering functions $I$. We start by expressing each $I$ function in terms of the bare vertex functions as in $\mathrm{Eq}$. (25). Then we express each of the vertex functions in scaling form, using the scaling law Eq. (28) and the necessary factors to convert from $\Gamma_{R}$ 's to $\Gamma_{b}$ 's as in $E q$. (27). The $I$ functions can then be simplified by combining some prefactors in $w, S_{2}$, etc. Finally, we eliminate $w$ and $y$ in favor of $N$ and the overlap $s$, using the implicit relations (37) and (38). The result is a scaling formula for each scattering function $I$ in which (for fixed overlap $s$ ) all the $N$ dependence has been made explicit. The dependence on $q, C_{p}$, and $N$ has been reduced to a scaling function $g$ with two variables only:

$I_{0}\left(q, C_{p}, N\right)=l^{-d} S_{2}^{-\nu d}\left(g^{*}\right)^{-1} N^{2-\nu d} g_{0}(Q, s)$,

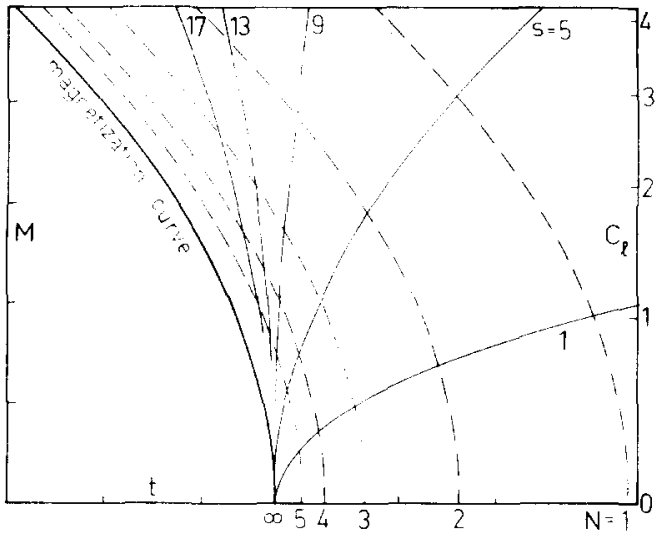

FIG. 5. Polymer variabies $N, C_{l}$, and $s$ plotted against the magnetic variables $M$ and $t$, using the mean field approximation.

$$
\begin{aligned}
C_{p} i_{a}\left(q, C_{p}, N\right) & =l^{-d} S_{2}^{-\nu d}\left(g^{*}\right)^{-1} N^{2-\nu d} \mathfrak{g}_{a}(Q, s), \\
\mathfrak{g}_{0(a)}(Q, s) \equiv & \left(\frac{f(y)}{2\left(\partial F^{(0,0)} / \partial y\right)}\right)^{2-\nu d} \\
& \times\left[\frac{\left[F_{(a)}^{(1,1)}(Q,-Q, y)\right]^{2}}{F_{(a)}^{(0,2)}(Q,-Q, y)}-F_{(a)}^{(2,0)}(Q,-Q, y)\right],
\end{aligned}
$$

where $y(s)$ is defined implicitly by Eq. (37) The endpoint scattering functions obey

$$
\begin{aligned}
& I_{e}\left(q, C_{p}, N\right)=4 C_{p}^{2} l^{d}\left(S_{2} N\right)^{\nu d} g^{*} g_{e}(Q, s), \\
& i_{e a}\left(q, C_{p}, N\right)=4 C_{p} l^{d}\left(S_{2} N\right)^{\nu d} g^{*} g_{e a}(Q, s), \\
& g_{e(a)}(Q, s) \equiv\left(\frac{f(y)}{2\left(\partial F^{(0,0)} / \partial y\right)}\right)^{\nu d}\left[F_{(a)}^{(0,2)}(Q,-Q, y)\right]^{-1} .
\end{aligned}
$$

These scaling laws for the $I$ functions are the central result of this work. Again, the renormalization symmetry is expressed in the structure of Eqs. (40), (41), (43), and (44). The explicit form of the scaling functions $g$ and the value of the exponent $\nu$ are more detailed properties of the renormalized field theory. These are universal and are uniquely (if implicitly) defined by the field theory, independently of details of the system, and they can be computed approximately. Thus, for example, in mean field theory

$$
\mathfrak{g}_{0}(Q, s)=\frac{1}{Q^{2}+1 /(2 s)+\frac{1}{3}}, \mathfrak{g}_{a}(Q, s)=\frac{1}{Q^{2}+1 /(2 s)} .
$$

The details of the system enter only in the scale factors $l$ and $S_{2}$.

We want to add some remarks on the range of validity of these equations. Above, we have stressed that we are concerned with length scales large compared to $l$ and $\Lambda^{-1}$. If the average distance between different links is to fulfill this condition we have to restrict ourselves to dilute solutions of very long chains. We therefore expect these results to be correct in the limit $N \gg 1$; $C_{l} \ll l^{-d}, \Lambda^{d} ;$ and $q \ll l^{-1}, \Lambda$ [which corresponds to the critical region $t \approx 0, M \approx 0$ (Fig. 5)] ${ }^{26 \mathrm{~b}}$ Experimentally it is found ${ }^{27}$ that in good solvents universal power laws hold for $N \gtrsim 100$ monomers and almost all concentrations.

The general laws have not yet been tested experimentally. Rather, experiments concentrate on certain lim- 
iting regions. In the next section we evaluate the general laws in some limits of interest. This can be done with the help of further information.

\section{SPECIAL LIMITS}

\section{A. Single chain limit}

To obtain this limit we assume the existence of a virial expansion, i.e., of an expansion for the free energy and expectation values in powers of $C_{p}$ for finite fixed $N$. From Eqs. (33), (35), and (22) we find for small $M_{0}^{2}$ and fixed $\zeta$ that $C_{p} \sim C_{l} \sim M_{0}^{2}$. Therefore the existence of the virial expansion is equivalent to the analyticity in $M_{0}^{2}$ of the vertex functions for $t>0$ (Griffiths analyticity). Obviously for $C_{b} \rightarrow 0$ the correlation functions tend to their autocorrelation parts and, starting with $M_{0}^{2}$ terms, both $I_{0}$ and $I_{e}$ are proportional to $C_{p}$ in that limit. We find

$$
\begin{aligned}
\lim _{c_{p} \rightarrow 0} \frac{1}{C_{p}} I_{0}\left(q, C_{p}, N\right)= & l^{-d} S_{2}^{-\nu d}\left(g^{*}\right)^{-1} N^{2-\nu d} \\
& \times\left(l^{d} g * S_{2}^{\nu d} N^{\nu d}\right) \lim _{s=0} \frac{1}{s} g_{0}(Q, s) \\
& \equiv N^{2} \hat{\jmath}_{0}\left[q l\left(N S_{2}\right)^{\nu}\right] .
\end{aligned}
$$

Performing the same steps on $I_{e}$, we recover a singlechain limit without a prefactor in $N$ :

$$
\lim _{C_{p} \rightarrow 0} \frac{1}{C_{p}} I_{e}\left(q, C_{p}, N\right)=4 \hat{g}_{e}\left[q l\left(N S_{2}\right)^{\nu}\right] \text {. }
$$

Defining the radius of gyration as the negative Laplacian of the logarithm of $I_{a}, R^{2}=-\left.\Delta_{q} \ln I_{a}\left(q, C_{p}, N\right)\right|_{q=0}$, we immediately find

$$
R^{2}\left(C_{p}=0\right)=l^{2}\left(N S_{2}\right)^{2 \nu}\left[-\Delta_{Q} \ln \hat{g}_{0}(Q)\right]_{Q=0},
$$

which matches the empirical scaling law mentioned in the introduction. In the next order of the virial expansion, we find scaling laws for the second virial coefficients; for instance, we may obtain the compressibility $I_{0}(q=0)$ :

$$
I_{0}\left(0, C_{p}, N\right)=C_{p} N^{2}\left[g_{0}^{(1)}+\frac{1}{2} C_{p} l^{d} g^{*}\left(S_{2} N\right)^{\nu d} g_{0}^{(2)}+\mathcal{O}\left(C_{p}^{2}\right)\right],
$$

where the universal numbers $g_{0}^{(1)}, g_{0}^{(2)}$ are derivable from $\mathfrak{s}_{0}(Q, s)$ [Eq. (42)]. Similarly, the correlation length $\xi$ of the full scattering function $I_{0}$ can be written

$$
\begin{aligned}
\xi & \equiv\left[-\left.\Delta_{q} \ln I_{0}\left(q, C_{p}, N\right)\right|_{\alpha=0}\right]^{1 / 2} \\
& =l\left(N S_{2}\right)^{\nu}\left[\xi^{(1)}+\frac{1}{2} C_{p} l^{d} g^{*}\left(S_{2} N\right)^{\nu d} \xi^{(2)}+\mathcal{O}\left(C_{p}^{2}\right)\right],
\end{aligned}
$$

where $\xi^{(1)}, \xi^{(2)}$ are also obtained from $g_{0}(Q, s)$, together with the definition of $Q$ [Eq. (39)]. The quantity $\frac{1}{2} N^{2} \mathfrak{g}_{0}^{(1)}$ represents the second moment $\left\langle N^{2}\right\rangle$ of the length distribution in our ensemble, as is easily seen from the definition of $I_{a}$. Using Eqs. (49) and (50) and similar relations for other quantities, we can define a number of experimentally accessible universal ratios. By way of example we mention one expression ${ }^{28}$ which can be determined from $I_{0}\left(q, C_{p}, N\right)$ alone:

$$
\xi^{-d}\left(C_{p}=0\right)\left[\frac{\left(\partial^{2} / \partial C_{p}^{2}\right) I_{0}}{\left(\partial I_{0} / \partial C_{p}\right)}\right]_{q=C_{p}=0}=\left(\xi^{(1)}\right)^{-d} \frac{g^{*} g_{0}^{(2)}}{g_{0}^{(1)}} .
$$

In the $(4-d)(\equiv \epsilon)$ expansion this has the value $-\epsilon \pi^{2} / 8$
$+\mathcal{O}\left(\epsilon^{2}\right)$, as can be deduced from Eqs. (27"), (40), and (45a).

\section{B. Limit of large $s$}

We may realize the limit of large $s$ by letting $N \rightarrow \infty$ as $C_{l}$ remains constant, thereby preserving the condition that $C_{l}^{-d} \gg l$. This is known as the semidilute regime. A general statement of the behavior of the correlation function for the $n=0$ model has not yet been found. In the limit $N \rightarrow \infty$ we approach the magnetization curve (see Fig。 5) where for $n \geq 2$ new (Goldstone) singularities are found. With convincing argument missing, we make the simplest possible hypothesis. We assume that no new dominant singularity appears at the magnetization curve, and that the leading power of $N$ in the limit $N \rightarrow \infty, C_{l} \neq 0$, fixed, is given by mean field theory, Eq. $\left(45^{\prime}\right)_{0}^{29}$ This implies for instance that $I_{0}\left(q, C_{p}, N\right)$ tends to some finite value

$I_{0}\left(q, C_{p}, N\right) \rightarrow C_{l} S_{2}^{-1}\left(C_{l} S_{2} l^{d} g^{*}\right)^{1 /(1-\nu d)} g_{\infty}\left[q l\left(C_{l} S_{2} l^{d} g^{*}\right)^{\nu /(1-\nu d)}\right]$,

where

$$
g_{\infty}\left[q l\left(C_{l} S_{2} l^{d} g^{*}\right)^{\nu /(1-\nu d)}\right]=\lim _{\substack{N \rightarrow \infty \\ C_{l} \text { fixed }}}(s)^{(2-\nu d) /(\nu d-1)} \mathcal{G}_{0}(Q, s) 。
$$

Thus

$$
I_{0}\left(0, C_{p}, N\right)-C_{l} S_{2}^{-1}\left(C_{l} S_{2} l^{d} g^{*}\right)^{1 /(1-\nu d)} g_{\infty}(0)
$$

and

$$
\xi \rightarrow l\left(C_{l} S_{2} l^{d} g^{*}\right)^{\nu /(1-\nu d)}\left[-\left.\Delta_{k} \ln g_{\infty}(k)\right|_{k=0}\right]^{1 / 2} .
$$

The power laws for $I_{0}\left(0, C_{p}, N\right)$ and $\xi$ have first been given by Daoud et al. ${ }^{14}$ They are supported by experiment. Again we can construct universal ratios like

$$
\begin{aligned}
\frac{\xi^{-d} I_{0}\left(0, C_{p}, N\right)}{C_{l}^{2}} & \rightarrow g * g_{\infty}(0)\left[-\left.\Delta_{k} \ln g_{\infty}(k)\right|_{0}\right]^{-d / 2} \\
& \rightarrow \frac{1}{8} \pi^{2} \epsilon+\mathcal{O}\left(\epsilon^{2}\right) .
\end{aligned}
$$

Results for the other scattering functions can be derived in a corresponding fashion.

\section{Behavior for large momentum transfer}

For momenta in the range $\xi^{-1} \ll q \ll \Lambda$ the behavior of the scattering functions can be studied by field-theoretic methods. We use Weinberg's theorem ${ }^{30}$ on the largemomentum behavior of Feynman diagrams to identify those diagrams which contribute to the leading term. We prove in Appendix B. I that the leading diagrams in $I_{0}\left(q, C_{p}, N\right)$ are of the type of autocorrelation diagrams. We therefore conclude that $I_{0}$ and $C_{p} i_{a}$ become equal in this limit:

$$
I_{0}\left(q, C_{p}, N\right)=C_{p} i_{a}\left(q, C_{p}, N\right)\left[1+\vartheta\left(\frac{q}{\Lambda}, \frac{1}{q \xi}\right)\right] .
$$

The interpretation of this result is obvious. It implies that in a semidilute solution there exists a range of distances large compared to the scale of the microscopic structure, where still the probability of finding two monomers on the same chain dominates that for finding two monomers on different chains.

The asymptotic behavior of $I_{0}\left(q, C_{p}, N\right)$ can be worked out with the help of the short-distance expansion. ${ }^{31}$ This method assumes that a product of two operators local- 
ized at neighboring points in space can be expanded in a sum of local operators. We give an example:

$\varphi^{2}(r-x / 2) \varphi^{2}(r+x / 2)=c_{1}(x) \varphi^{2}(r)+c_{2}(x) \varphi^{4}(r)+\cdots \cdot$

The coefficients $c_{i}(x)$ are number-valued functions. In Appendix B. II we use this method to find the asymptotic behavior of $I_{0}\left(q, C_{p}, N\right)$ :

$$
\begin{aligned}
I_{0}\left(q, C_{p}, N\right) \sim & a_{1} S_{2}^{-1} C_{l}(l q)^{-1 / \nu} \\
& +a_{2}\left[\left(\frac{\partial F^{(0,0)}}{\partial y}\right)^{-1} C_{l} l^{d} g^{*} S_{2}\right]^{2 \beta /(\nu d-1)}(q l)^{(\gamma-2) / \nu},
\end{aligned}
$$

where $a_{1}$ and $a_{2}$ are undetermined numerical coefficients, and $\gamma \equiv \nu d-2 \beta$. The exponents of both terms are approximately equal, the next corrections being about a factor of $q^{-2}$ smaller. The coefficients $a_{1}$ and $a_{2}$ can be calculated from renormalized perturbation theory, where $a_{2}$ is found to vanish in lowest order.

The large momentum behavior of the end-point correlation functions is well known. ${ }^{32}$ We here recall the result, expressed in polymer variables:

$$
\begin{aligned}
C_{p} i_{e a}\left(q, C_{p}, N\right) & \sim I_{e}\left(q, C_{p}, N\right) \\
& \sim 4 C_{p}^{2} l^{d} g^{*}\left(N S_{2} \frac{f(y)}{2\left(\partial F^{(0,0)} / \partial y\right)}\right)^{2 \beta} a_{3}(q l)^{-\gamma / \nu} .
\end{aligned}
$$

\section{CONCLUSION}

We have extended the renormalized theory of a polymer solution to include the experimentally measured scattering functions. The theory predicts the scaling laws of Eqs. (40), (41), (43), and (44), which constrain the dependence of the scattering on $q, C_{p}$, and $N$. The field theory also allows us to infer the behavior of the scaling functions $\boldsymbol{g}(Q, s)$ for large or small $Q$ and for small overlap $s$. We have also suggested a plausible behavior for large $s$. Using these results we obtained the power laws of Eqs. (48)-(50) for the compressibilities and correlation lengths for small overlap. At large overlap these quantities are given by Eqs. $\left(53^{\circ}\right)$ and (54). The leading power behavior for all these equations has been verified experimentally. ${ }^{11}$ The dominant behavior at high $q$ is predicted in Eq. (58). This is also consistent with experiments. ${ }^{27}$ Reference $10 \mathrm{~b}$ even hints that the second term in Eq. (58) may be visible. Beyond these leading power laws, the field theory can predict the functional form of the scaling functions $g_{\text {. Once the }}$ $g$ are calculated, they can be compared with experiment by measuring dimensionless ratios such as Eqs. (51) and (55). Our calculation of the $g$ functions is in progress.

\section{ACKNOWLEDGMENTS}

The authors owe a great debt to $\mathrm{E}$. Brézin and the Service de Physique Théorique at Saclay for hospitality, guidance, and support (of T.W.). L. S. wants to thank the Deutsche Forschungsgemeinschaft for a fellowship. We benefited from discussions with M. Mirkovitch, G。 Jannink, M. Daoud, J. Des Cloizeaux, and Royce K. P. Zia.

\section{APPENDIX A: FIELD-THEORETIC REPRESENTATION OF THE SCATTERING FUNCTIONS}

We prove that with the definitions [cf.Eqs. (4) and (14)]

$$
\begin{aligned}
& H=H_{0}+H_{I}+\int d^{d} x \zeta(x) \rho(x), \\
& \mathcal{L}[\varphi, \zeta]=L[\varphi]+\frac{1}{2 l^{2}} \int d^{d} x \zeta(x) \varphi^{2}(x),
\end{aligned}
$$

we have

$$
Z[H]=\lim _{n \rightarrow 0} \int \prod_{\alpha=1}^{n} d\left[\varphi_{\alpha}(x)\right] e^{-\mathcal{L}[v, 5]} .
$$

The basic tool is the following lemma ${ }^{5}$ We define

$$
\begin{aligned}
& \mathscr{S}\left(N ; q_{0}, \cdots, q_{r} ; j_{1}, \cdots, j_{r-1}\right)=\left(4 l^{2} \pi\right)^{-N(d / 2)} \int d^{d} x_{0} \cdots d^{d} x_{N} \exp \left(-i \sum_{\rho=0}^{r} q_{\rho} x_{f_{p}}-\frac{1}{4 l^{2}} \sum_{\eta=1}^{N}\left(x_{\eta}-x_{\eta-1}\right)^{2}\right), \\
& 0=j_{0}<j_{1} \leq \ldots \leq j_{r-1} \leq j_{r}=N .
\end{aligned}
$$

It is found by Gaussian integration that

$$
g\left(N ; q_{0}, \cdots, j_{r-1}\right)=(2 \pi)^{d} \delta^{d}\left(\sum_{\rho=0}^{r} q_{\rho}\right) \exp \left[-l^{2} \sum_{\rho=0}^{r-1}\left(\sum_{\sigma=0}^{\rho} q_{\sigma}\right)^{2}\left(j_{\rho+1}-j_{\rho}\right)\right] .
$$

We now sketch the proof of Eq. (A2), using the one-chain contribution,

$$
\left.A \equiv \frac{\partial Z[H]}{\partial l^{2} h_{0}^{2} / 2}\right|_{h_{0}=0}
$$

as an example:

$$
A=l^{-d} \sum_{N=1}^{\infty} e^{-\zeta N} \int d^{d} x_{0} \cdots d^{d} x_{N} \exp \left(-\frac{1}{4 l^{2}} \sum_{\eta=1}^{N}\left(x_{\eta}-x_{\eta-1}\right)^{2}-\frac{1}{2} \sum_{j, j^{\prime}=1}^{N} V\left(x_{j}-x_{j^{\prime}}\right)-\sum_{\lambda=1}^{N} \zeta\left(x_{\lambda}\right)\right)\left(4 l^{2} \pi\right)^{-N(d / 2)} .
$$


We write

$$
V(x)=\int d^{d} q \tilde{V}(q) e^{-i q x}, \quad \zeta(x)=\int d^{d} q \tilde{\zeta}(x) e^{-i q x},
$$

and we expand in powers of $\tilde{V}$ and $\tilde{\zeta}$ :

$$
A=l^{-d} \sum_{\alpha=0}^{\infty} \sum_{\beta=0}^{\infty} \frac{(-)^{\alpha+\beta}}{\alpha ! \beta ! 2^{\alpha}} \int d^{d} q_{1} \cdots d^{d} q_{2 \alpha+\beta} \prod_{\gamma=1}^{\alpha} \tilde{V}\left(q_{\gamma}\right) \delta^{d}\left(q_{\gamma}+q_{\alpha+\gamma}\right) \prod_{\delta=1}^{\beta} \tilde{\zeta}\left(q_{2 \alpha+\sigma}\right) \hat{\boldsymbol{g}}\left(\zeta, q_{1} \cdots q_{2 \alpha+\sigma}\right),
$$

where

$$
\hat{\mathfrak{g}}\left(\zeta, q_{1}, \cdots, q_{2 \alpha+\beta}\right) \equiv \sum_{N=1}^{\infty} e^{-\zeta N} \sum_{j_{1} \cdots j_{2 \alpha+\beta}=1}^{N} \int d^{d} x_{0} \cdots d^{d} x_{N}\left(4 l^{2} \pi\right)^{-N(d / 2)} \exp \left(-\frac{1}{4 l^{2}} \sum_{\rho=1}^{N}\left(x_{\rho}-x_{\rho-1}\right)^{2}-i \sum_{\rho=1}^{2 \alpha+\beta} q_{\rho} x_{j_{\rho}}\right) \cdot
$$

Ordering the $j$ 's and applying the lemma with $q_{0}=q_{r}=0$, we find

$$
\hat{\mathfrak{g}}\left(\zeta ; q_{1} \cdots q_{2 \alpha+\beta}\right)=\sum_{P\{2 \alpha+\beta\}} \sum_{N=0}^{\infty} \sum_{0<j_{1} \leqslant \cdots \leqslant j_{2 \alpha+\beta} \leqslant N}(2 \pi)^{d} \delta^{\alpha}\left(\sum q_{\rho}\right) \exp \left\{-\sum_{\rho=0}^{2 \alpha+\beta}\left[\zeta+l^{2}\left(\sum_{\sigma=0}^{\rho} q_{P(\sigma)}\right)^{2}\right]\left(j_{\rho+1}-j_{\rho}\right)\right\},
$$

where the first sum ranges over all permutations $P$ of $\{1,2, \cdots,(2 \alpha+\beta)\}$. Next we transform the summations into integrals, assuming that both $\zeta$ and $l^{2} q_{\sigma}^{2}$ are small. This yields

$$
\hat{\mathfrak{g}}\left(\zeta, q_{1} \cdots q_{2 \alpha+\beta}\right)=(2 \pi)^{d} \delta^{d}\left(\sum q_{\rho}\right) \sum_{P} \prod_{\rho=0}^{2 \alpha+\beta}\left[\zeta+l^{2}\left(\sum_{\sigma=0}^{\rho} q_{P(\sigma)}\right)^{2}\right]^{-1},
$$

or

$$
\begin{aligned}
A= & l^{-d}(2 \pi)^{d} \sum_{\alpha, \beta} \frac{(-)^{\alpha+\beta}}{2^{\alpha} \alpha ! \beta !} \sum_{p\{2 \alpha+\beta\}} \int d^{d} q_{0} \cdots d^{d} q_{2 \alpha+\beta} \delta^{d}\left(\sum_{\rho=0}^{2 \alpha+\beta} q_{\rho}\right) \delta^{d}\left(q_{0}\right) \prod_{\gamma=1}^{\alpha}\left(\frac{1}{l^{4}} \tilde{V}\left(q_{P(\gamma)}\right) \delta^{d}\left(q_{P(\gamma)}+q_{P(\alpha+\gamma)}\right)\right) \\
& \times \prod_{\delta=1}^{\beta}\left(\frac{1}{l^{2}} \tilde{\zeta}\left(q_{P(2 \alpha+\delta)}\right)\right) \prod_{\rho=0}^{2 \alpha+\beta}\left[(\zeta) l^{-2}+\left(\sum_{\sigma=0}^{o} q_{\sigma}\right)^{2}\right]^{-1} \cdot
\end{aligned}
$$

This expression can be represented by the sum of all Feynman diagrams with one polymer line, propagators $\left[(\zeta) / l^{2}+q^{2}\right]^{-1}$, four-point vertices of the type $l^{-4} \tilde{V}(q)$, and a two-point vertex $\bar{\zeta}(q) l^{-2}$. This concludes our sketch of the proof of Eq. (A2).

To include autocorrelations, we introduce a second $n$-component field $\varphi^{\prime}$ into the Lagrangian, with its own $h_{0}^{\prime}$ and $\zeta^{\prime}(x)$ parameters. The $\varphi^{\prime}$ field interacts exactly like the original $\varphi$ field; thus the Lagrangian $\tilde{\mathbb{L}}$ for the whole system becomes

$$
\overline{\mathscr{L}}=L\left[\varphi, \zeta, h_{0}\right]+L\left[\varphi^{\prime}, \zeta^{\prime}, h_{0}^{\prime}\right](2 \pi)^{d} l^{-4} \int d^{d} x \int d^{d} x^{\prime}\left|\varphi^{\prime}(x)\right|^{2} V\left(x-x^{\prime}\right)\left|\varphi\left(x^{\prime}\right)\right|^{2}
$$

The autocorrelation $\left\langle\sum_{i} \rho_{i}(x) \rho_{i}\left(x^{\prime}\right)\right\rangle$ can then be expressed as

$$
\lim _{h_{0}^{\prime} \rightarrow 0} \lim _{n \rightarrow 0}\left[\frac{h_{0}^{2}}{h_{0}^{\prime 2}} \frac{\delta \Omega W_{n}[\tilde{\mathcal{L}}]}{\delta \zeta^{\prime}(x) \delta \xi^{\prime}\left(x^{\prime}\right)}\right] \text {. }
$$

\section{APPENDIX B: CORRELATION FUNCTIONS FOR LARGE MOMENTUM}

\section{Proof of Eq. (56)}

The proof is based on Weinberg's theorem. ${ }^{30}$ The asymptotic behavior of a diagram $D(q)$ up to $\log (q)$ factors is given by $D(q) \sim q^{\Sigma(w)}$, where $\Sigma(w) \equiv \sup _{s \in D} w(s)$ and $w(s) \equiv d L(s)-2 l(s)$. Here $L(s)$ and $l(s)$ are the number of loops and the number of internal lines of the subdiagram $s$. The symbol $s$ represents any connected subdiagram of $D$ which contains all external vertices with nonvanishing momentum (essential subdiagrams).

Let $D_{a}[D]$ denote the set of all diagrams contributing to $\Gamma_{a}^{(2,0)}\left[\Gamma^{(2,0)}\right]: D=D_{a} U\left(D-D_{a}\right)$. An essential subdiagram contains both $\varphi^{2}$ insertions and has $E$ external legs. Using well-known topological relations we find

$$
-w(s)=E(s)+(4-d) L(s) .
$$

The leading contribution to $D_{a}$ arises from $E=2$. All essential subdiagrams of $D$ in $\left(D-D_{a}\right)$ have $E=4$. Therefore the large $-q$ contribution from $D-D_{a}$ is dominated by that of $D_{a}$. As usual, the argument holds order for order in the loop expansion. This proves our statement for the irreducible part. The reducible part can be treated in the same way.

\section{The large-momentum behavior of $I_{0}\left(q, C_{p}, N\right)$}

Treating $\Gamma_{R}^{(1,1)}(q,-q, t, M)$ first, we apply the operator product expansion in the form

$\varphi_{\alpha}(q)|\varphi|^{2}(-q)=c_{1}(q, \mu) \varphi_{\alpha}(0)+c_{3}(q, \mu) \varphi_{\alpha}(0)|\varphi(0)|^{2}$.

Substituting this ansatz into $\Gamma_{R}^{\left(L, N^{\prime}\right)}(q, 0, \cdots 0 ;-q, 0$, $\ldots 0 ; t)$ we find

$$
\begin{gathered}
\Gamma_{R}^{\left(L, N^{\prime}\right)}(q, 0 \cdots 0 ;-q, 0 \cdots 0 ; t)=c_{1}(q, \mu) \Gamma_{R}^{\left(L-1, N^{\prime}\right)}(0 \cdots 0 ; t) \\
+c_{3}(q, \mu) \Gamma_{\varphi^{3}, R}^{\left(L-1, N^{\prime}-1\right)}(0 \cdots 0 ; t) .
\end{gathered}
$$


The equations of motion ${ }^{17}$ for a $\varphi^{4}$ theory show that $\Gamma_{\varphi^{3}, R}^{\left(L-1, N^{\prime}-1\right)}(0 \cdots 0 ; t)$ is proportional to $\Gamma_{R}^{\left(L-1, N^{\prime}\right)}(0 \cdots 0 ; t)$, and we therefore find

$$
\begin{array}{r}
\Gamma^{\left(L, N^{*}\right)}(q, 0 \cdots 0 ;-q, 0 \cdots 0 ; t) \\
\quad=c(q, \mu) \Gamma^{\left(L-1, N^{\prime}\right)}(0 \cdots 0 ; t) .
\end{array}
$$

Use of the scaling laws for $M=0$ yields

$$
\mu^{2}\left(\frac{t}{\mu^{2}}\right)^{-1} \hat{F}^{\left(L, N^{\prime}\right)}\left[\frac{q}{\mu}\left(\frac{t}{\mu^{2}}\right)^{-\nu}\right]=c(q, \mu) \hat{F}^{\left(L-1, N^{\prime}\right)}(0),
$$

or

$$
c(q, \mu)=a \mu^{-2}\left(\frac{q}{\mu}\right)^{-1 / \nu} .
$$

Equations (B3) and (B4) hold only if primitively divergent vertex functions do not appear on the right-hand side; this excludes $N^{\prime}=4, L \leq 1$ and $N^{\gamma}=2, L \leq 2$. To evaluate $\Gamma^{(1,4)}$ and $\Gamma^{(1,2)}$ we increase $L$ by differentiating with respect to $t$, apply Eqs. (B5) and (B6), and integrate back. The integration constants give rise to additional terms, which have to be analyzed with the help of the scaling laws. We find

$$
\begin{aligned}
& \Gamma_{R}^{(1,4)}(q ;-q, 0,0,0 ; t) \\
& \quad=c(q, \mu) \Gamma_{R}^{(0,4)}(0 \cdots \cdot 0 ; t)+\mu^{2-d} a_{1}\left(\frac{q}{\mu}\right)^{(1 / \nu)(2 \gamma-\nu d-1)}, \\
& \Gamma_{R}^{(1,2)}(q ;-q, 0 ; t) \\
& \quad=c(q, \mu) \Gamma_{R}^{(0,2)}(0,0 ; t)+a_{2}\left(\frac{q}{\mu}\right)^{(\gamma-1) / \nu}+a_{3}\left(\frac{q}{\mu}\right)^{(\gamma-2) / \nu} .
\end{aligned}
$$

Substituting these results into $\mathrm{Eq}$. (22), we find after some straightforward manipulations

$$
\begin{aligned}
F^{(1,1)}(Q,-Q, y)= & a_{1}^{(1,1)} Q^{(\gamma-1) / \nu}+a_{2}^{(1,1)} f(y) Q^{-1 / \nu} \\
& +a_{3}^{(1,1)} Q^{(1 / \nu)(2 \gamma-\nu d-1)}+a_{4}^{(1,1)} y Q^{(\gamma-2) / \nu} .
\end{aligned}
$$

The asymptotic behavior of $\Gamma_{R}^{(0,2)}$ can be taken from the literature. ${ }^{32}$ For the discussion of $\Gamma_{R}^{(2,0)}$ we use the relation

$$
\varphi^{2}(q) \varphi^{2}(-q) \rightarrow C_{2}(q, \mu) \varphi^{2}(0) .
$$

Proceeding in the same way as above, we find

$$
\begin{aligned}
F^{(2,0)}(Q,-Q, y)= & a_{1}^{(2,0)} \frac{\partial F^{(0,0)}}{\partial y} Q^{-1 / \nu}+a_{2}^{(2,0)} Q^{(\gamma-2) / \nu} \\
& +n a_{3}^{(2,0)} y Q^{(1 / \nu)(\nu d-3)}+n a_{4}^{(2,0)} Q^{(1 / \nu)(\nu d-2)} .
\end{aligned}
$$

The last two terms vanish in the limit $n \rightarrow 0$. Note that these results can also be derived with the help of the Callan-Symanzik equations for a massive theory.

\footnotetext{
${ }^{1}$ (a) See, for example, H. Yamakawa, Modern Theory of Polymer Solutions (Harper and Row, New York, 1970); (b) Thus we do not consider here the observed variations in $\nu$ near the collapse temperature $\theta$.
}

${ }^{2}$ D. S. McKenzie and M. A. Moore, J. Phys. A 4, 182 (1971).
${ }^{3}$ P. J. Flory, Principles of Polymer Chemistry (Cornell U. P. . Ithaca, NY, 1970).

${ }^{4} \mathrm{P}$. G. DeGennes, Phys. Lett. A 38, 339 (1972).

${ }^{5} \mathrm{~J}$. Des Cloizeaux, J. Phys. (Paris) 36, 281 (1975).

${ }^{6}$ K. G. Wilson and J. Kogut, Phys. Rep, C 12 (1974).

${ }^{7}$ V. J. Emery, Phys. Rev. B 11, 239 (1975)

${ }^{8}$ A. Aharony, Y. Imry, and S. K. Ma, Phys, Rev. B 13, 466 (1976).

${ }^{9}$ P. W. Kasteleyn and C. M. Fortuin, J. Phys. Soc. Jpn. Suppl. 26, 11 (1969); A. B. Harris et al. . Phys. Rev. Lett. 35,327 (1975); 35, 1397 (E) (1975).

${ }^{10}$ (a) A. B. Harris, T. C. Lubensky, and J. - H. Chen, Phys. Rev. Lett. 36, 415 (1976). (b) J. Mazur and D. McInty re. Macromolecules 8, 464 (1975).

${ }^{11}$ M. Daoud et al. , Macromolecules 8, 804 (1975).

${ }^{12}$ S. F. Edwards, Proc. Phys. Soc. London 88, 265 (1966).

${ }^{13}$ K. G. Wilson, Phys. Rev. 179, 1499 (1969).

${ }^{14}$ L. P. Kadanoff, Phys. Rev. Lett. 23, 1430 (1969).

${ }^{15} \mathrm{~J}$. Des Cloizeaux, Phys. Rev. A 10, 1665 (1974): H. J. Hilhorst, Phys. Lett. A 56, 153 (1976).

${ }^{16}$ E. Brezin, J. C. LeGuillou, and J. Zinn-Justin. Phys. Rev。 D 8, 434 (1973).

${ }^{17}$ E. Brézin, J. C. LeGuillou, and J. Zinn-Justin, in Phase Transitions and Critical Phenomena, edited by C. Domb and M. S. Green (Academic, New York, to be published), Vol. VI.

${ }^{18}$ For similar models, see Ref. 11 and K. F. Freed, Adv. Chem. Phys. 22, 1 (1972).

${ }^{19}$ The potential may have attractive parts as well, provided they are sufficiently weak. The attraction between links in real polymers is believed to be "sufficiently weak" provided the temperature is above the collapse temperature $\theta$. See P. G. DeGennes, J. Phys. (Paris) Lett, 36, L55 (1975).

${ }^{20}$ See, for example, C. Bloch, Stud. Stat. Mech. 3, 1. (1965).

${ }^{21}$ The resulting diagrammatic structure is discussed, for example, in Freed, Ref. 18, p. 74.

${ }^{22}$ The interchange of this limit with the differentiation is justified to any order in perturbation theory, since any diagram has the form (polynomial in $n$ ) times a well-behaved function of $\zeta$.

${ }^{23}$ See Ref. 17. See also C. De Dominicis, J. Math. Phys. 4, 225 (1963); C. De Dominicis and P. C. Martin, J. Math. Phys. 5, 14, 31 (1964); G. Jona-Lasinio, Nuovo Cimento 34, 1790 (1964).

${ }^{24}$ We use zero-mass renormalization, and we take notation and results from the review paper of Ref. 17 .

${ }^{25} \mathrm{E}$. Brezin, J. C. Le Guillou, J. Zinn-Justin, Phys. Rev. D 8,2418 (1973)。

${ }^{26}$ (a) P. J. Flory, Principles of Polymer Chemistry (Cornell, U. P. , Ithaca, NY, 1967), 6thed., Chap. XIV. (b) For $N$ sufficiently small or $C_{l}$ sufficiently large we expect departures from the scaling laws (40), (41), (43), and (44). By adapting the results of Ref. 25 one finds that these departures from the scaling laws are of relative order $N^{-\omega \omega}, C_{l}^{-\nu \omega /\left(\omega_{t-1}\right)}$, and $q^{\omega}$. The exponent $\omega$ is estimated to lie between 0.5 and 1 .

${ }^{27}$ B. Farnoux, Ann. Phys. (Paris) 1, 73 (1976).

${ }^{28}$ The universal ratios will depend on the length distribution of the ensemble. We hope to study this effect in a further paper.

${ }^{29}$ It is not clear whether this hypothesis is compatible in all cases with the $\epsilon$-expansion results.

${ }^{30}$ s. Weinberg, Phys. Rev. 118, 838 (1960).

${ }^{31} \mathrm{~K}$. G. Wilson and W. Zimmermann, Commun. Math. Phys. 24, 87 (1972).

${ }^{32}$ E. Brezin, C. De Dominicis, and J. Zinn-Justin, Lett. Nuovo Cimento 10, 849 (1974).

${ }^{33}$ The derivation is easily modified for $\zeta$ not small. 